\title{
We need to target trauma: A prospective observational study in Eastern Cape Province, South Africa
}

\author{
K Clark, ${ }^{1}$ BA; A Rao, ${ }^{2}$ MSPH; V Chen, ${ }^{3}$ BA; P Mda, ${ }^{4}$ MBBS; F Piek, ${ }^{5}$ MBBS; J Irudeo, ${ }^{6}$ MBBS; R Maharaj, ${ }^{7}$ MBBS; L Wallis, ${ }^{8}$ MB ChB, MD; \\ T Quinn, ${ }^{9,10} \mathrm{MD}, \mathrm{PhD} ;$ B Hansoti, ${ }^{2,11} \mathrm{BSc}, \mathrm{MPH}, \mathrm{MB} \mathrm{ChB}, \mathrm{PhD}$ \\ ${ }^{1}$ Johns Hopkins University School of Medicine, Baltimore, Md, USA \\ ${ }^{2}$ Department of International Health, Johns Hopkins Bloomberg School of Public Health, Baltimore, Md, USA \\ ${ }^{3}$ Krieger School of Arts and Sciences, Johns Hopkins University, Baltimore, Md, USA \\ ${ }^{4}$ Nelson Mandela Academic Clinical Research Unit, Mthatha, Eastern Cape, South Africa \\ ${ }^{5}$ Department of Emergency Medicine, Frere Hospital, East London, South Africa \\ ${ }^{6}$ Department of Family Medicine, Mthatha Regional Hospital, Mthatha, South Africa \\ ${ }^{7}$ Department of Emergency Medicine, Livingstone Hospital, Port Elizabeth, South Africa \\ ${ }^{8}$ Division of Emergency Medicine, Faculty of Health Sciences, University of Cape Town, South Africa \\ ${ }^{9}$ Department of Medicine, Johns Hopkins University School of Medicine, Baltimore, Md, USA \\ ${ }^{10}$ Division of Intramural Research, National Institute of Allergy and Infectious Diseases, National Institutes of Health, Bethesda, Md, USA \\ ${ }^{11}$ Department of Emergency Medicine, Johns Hopkins University School of Medicine, Baltimore, Md, USA
}

Corresponding author: K Clark (kclark54@jhmi.edu)

Background. Emergency medicine accounts for a large proportion of medical care in many low- and middle-income countries. A better understanding of the burden of disease will guide training and resource allocation priorities, but lack of electronic medical records and standardised data collection systems makes it difficult to obtain this information.

Objectives. To draw attention to the proportionally large burden of trauma in emergency centres (ECs) throughout Eastern Cape Province, South Africa (SA), in the hope of influencing resource allocation and medical provider training protocols accordingly.

Methods. A secondary data analysis was performed from information gathered in HIV testing studies in two large tertiary care centres and one regional hospital in the Eastern Cape region of SA. All patients presenting to the ECs during the 6-week study period who met the inclusion criteria were approached and requested to provide consent for point-of-care HIV testing and collection of demographic information. Information collected included patient demographics, presenting complaints and final diagnoses. Simple descriptive statistics were used to analyse the data. Results. Data were collected from 4271 patients across three study sites: Frere Hospital ( $n=2$ 391), Nelson Mandela Academic Hospital $(n=622)$ and Mthatha Regional Hospital $(n=1258)$. At the two tertiary care centres, most patients were between the ages of 18 and 30 years (41.2\% and $32.6 \%$, respectively) and male (57.8\% and $60.2 \%$ ), and $70.4 \%$ and $41.5 \%$ had traumatic injuries. The most common complaints were stab/gunshot wounds (18.3\% and $20.2 \%)$. At the district hospital, the majority of patients were female (57.2\%), $40.1 \%$ were between 18 and 30 years old, and $27.3 \%$ presented with traumatic injuries. Stab/gunshot wounds were the second most common complaint (7.2\%) after lower respiratory tract infections (8.7\%).

Conclusions. From the proportion of presenting individuals sampled, we can conclude that a large proportion of care delivered in ECs in the Eastern Cape is for trauma. Local clinical capacitation efforts must focus on trauma training.

S Afr Med J 2020;110(1):38-43. https://doi.org/10.7196/SAMJ.2020.v110i1.13886

The ability to provide quality acute care is of global importance. According to the Disease Control Priorities project, implementation of acute care services could address $54 \%$ of the 45 million deaths in low- and middle-income countries (LMICs) each year. ${ }^{[1]}$ This is in contrast to high-income countries, where the top 10 leading causes of death are chronic conditions with common acute decompensations (e.g. Alzheimer's disease, cancers) and nonemergency conditions. ${ }^{[2]}$ A 2015 systematic review of acute care in 59 LMICs found that the sub-Saharan African acute care setting has significantly higher mortality rates than corresponding primary care clinics, and nearly 10 times the patient load. ${ }^{[3]}$ Understanding the burden of disease in an LMIC setting is critical to building acute care capacity, advocating for funding, developing training protocols and allocating resources.

Efforts to understand and improve acute care in LMICs have been delayed by lack of information on the services provided by emergency centres (ECs) in these regions. ${ }^{[2]}$ Specific informational needs that have been identified include better understanding of the conditions that drive patients to utilise emergency services, identification of effective, validated interventions in high-income settings and their adaptation to low-income settings, and advances in data collection methods for research and quality improvement. ${ }^{[3]}$ Notably, documentation of the chief complaint in acute care is of paramount importance, as providers operate on the basis of patients' self-reported issues in addition to cardinal findings on examination to develop a working diagnosis. ${ }^{[4]}$ However, under-documentation of the accurate range of acute presentations to health facilities in sub-Saharan Africa was identified by the 2011 African Federation for Emergency Medicine Consensus Conference as a critical barrier to the dissemination of targeted acute care. ${ }^{[5]}$

\section{Objectives}

To characterise the burden of disease across a diverse range of ECs in Eastern Cape Province, South Africa (SA). 


\section{Methods}

This was a secondary data analysis of a cross-sectional observational HIV testing study conducted in three ECs in the Eastern Cape (also known as the WISE study). All three study sites enrolled participants for a period of 6 weeks: September - November 2016 at Frere Hospital, East London; June - August 2017 at Nelson Mandela Academic Hospital, Mthatha; and July - August 2017 at Mthatha Regional Hospital. All study sites followed the study protocol outlined in the published analysis of the Frere Hospital study. ${ }^{[6]}$ All patients aged $>18$ years and not critically ill (critically ill was defined as a SA triage score of 'red') were eligible for enrolment. Data were prospectively collected by trained HIV counsellors, who captured demographic information and provider-initiated HIV testing acceptance and results.

\section{Study setting and participants}

This multisite study took place in three large hospitals in the Eastern Cape. The first study site was Frere Hospital, located in the urban city of East London. Frere Hospital is a tertiary care centre that serves nearly 3 million individuals in a catchment area of $>80000 \mathrm{~km}^{2}$ and has a 22-bed EC. The second study site was Nelson Mandela Academic Hospital, located in the rural city of Mthatha. It is also a tertiary care centre, serving $\sim 2.5$ million individuals in the Transkei region, which covers a total area of $45000 \mathrm{~km}^{2}$, and has a 15-bed EC. The third study site was Mthatha Regional Hospital, also located in the rural city of Mthatha. This hospital is a secondary care centre that serves the King Sabata Dalindyebo Local Municipality subdistrict of the Oliver Reginald Tambo District Municipality, covering a total area of just over $3000 \mathrm{~km}^{2}$. It serves 500000 individuals and has an 8-bed EC. None of these sites have access to electronic health records, and patient tracking is recorded in handwritten logbooks.

It is important to note the pertinent similarities and differences between the three study sites. Both Frere Hospital and Nelson Mandela Academic Hospital are large tertiary care centres, with acute surgical services available. The regional hospital is staffed by family medicine practitioners and the other sites by medical officers. None of the doctors or nurses have formal training in emergency medicine or trauma management. All three study sites manage cardiac, trauma, medical, surgical, and obstetrics and gynaecology patients in the same initial acute care setting before they are moved to a more specialised service.

\section{Data collection and analysis}

The data were prospectively collected at all three sites according to the WISE study protocol. Patients were asked their reason for the visit in their native language, and this information was verified with the healthcare provider, folder documentation, and nursing staff to enable accurate recording of the chief complaint. This strategy allowed us to accurately report data that would not be readily available via chart review methods that are more commonly used. Data collected included age, sex, chief complaint, vital signs, time of arrival, method of arrival and the subsequent final diagnosis. Patient information was collected on case report forms, which were scanned and entered using intelligent character recognition DataFax software (version 5.11) (Clinical DataFax Systems Inc., Canada) and centrally double-verified by independent data technicians. Chief complaints and final diagnoses were coded using the International Council for Harmonisation of Technical Requirements for Pharmaceuticals for Human Use (ICH)-developed MedDRA coding software (version 10.0), which allows for multiple levels of systematic categorisation. Results were analysed at the level of preferred term for chief complaints and system organ class for final diagnoses. Preferred- term categorisation refers to single medical concepts, whereas system organ class categorisation provides groupings for disease aetiologies. After all data were cleaned and validated, simple descriptive statistics were performed using Stata version 12 (StataCorp, USA). A limited number of data from Frere Hospital were not recorded and were accounted for in our analysis, although a formal sensitivity analysis was not performed.

\section{Ethical considerations}

The study was approved by the Johns Hopkins University School of Medicine Institutional Review Board (ref. no. IRB00105801), the University of Cape Town Human Research Ethics Committee (ref. no. 401/2013) and the Walter Sisulu University Human Research Ethics Committee (ref. no. 002/2016). Written consent was obtained from all participants who enrolled in the study and was required for both collection of demographic data and HIV testing.

Results from the study were shared with staff at Frere Hospital, Nelson Mandela Academic Hospital and Mthatha Regional Hospital through informal presentations.

\section{Results}

Data were collected from 4271 of a total of 13838 patients who presented to the three ECs during the study period. There were 2391 patients from Frere Hospital, 622 from Nelson Mandela Academic Hospital and 1258 from Mthatha Regional Hospital (Fig. 1).

\section{Frere Hospital}

Of the 2391 patients enrolled at this site, most were male ( $n=1360$, $56.9 \%)$. The median (interquartile range (IQR)) age was $33(26-45)$ years. Most patients had arrived via self-transport in a personal vehicle/taxi/bus ( $n=1577,66.0 \%)$ and with a new complaint $(n=2016$, $85.5 \%$ ), and visited the EC outside the normal hours of 09h00 - 17h00 ( $n=1277,53.4 \%$ ). Patients were triaged as emergency $(0.8 \%)$, very urgent (15.7\%), urgent (29.2\%) and routine (47.7\%), and 6.6\% did not

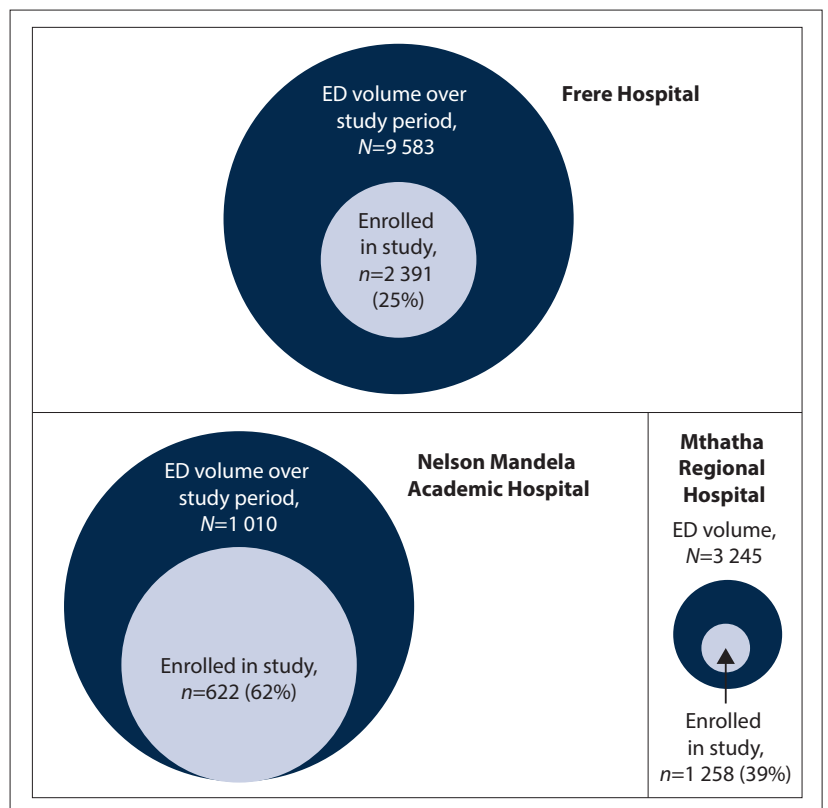

Fig. 1. Enrolment volume by study site. The entire box represents 6 million people, the combined catchment area of the three hospital sites. Individual boxes represent the catchment area for each hospital as a proportion of the whole (Frere Hospital 50\%, Nelson Mandela Academic Hospital 42\%, Mthatha Regional Hospital 8\%). (ED = emergency department.) 
receive a triage designation. The majority of the patients presented with trauma $(n=1656,69.3 \%)$ as opposed to medical complaints ( $n=697,29.2 \%)$ (Table 1).

The top five complaints reported were assault (14.2\%), stab wounds $(12.5 \%)$, falls $(10.0 \%)$, road traffic accidents $(9.8 \%)$ and abdominal pain $(4.6 \%)$. The top five final diagnoses were injury or poisoning (52.7\%), musculoskeletal and connective tissue disorders $(9.8 \%)$, infections and infestations $(8.2 \%)$, gastrointestinal disorders (4.6\%), and respiratory, thoracic and mediastinal disorders (4.1\%). Furthermore, trauma patients most commonly presented with stab/ cut injuries $(29.8 \%)$, a high proportion of which were in the extremities (59.4\%) (Table 2).

\section{Nelson Mandela Academic Hospital}

Of the 622 patients enrolled at this site, most were male ( $n=375$, $60.2 \%$ ). The median age was 32 years (IQR 25 -53). Most were referred from other hospitals or clinics $(n=406,65.3 \%)$, arrived via ambulance $(n=389,62.5 \%)$, and visited the EC outside the normal hours of 09 h00 - 17h00 $(n=389,62.5 \%)$. Patients were triaged as very urgent $(29.9 \%)$, urgent $(64.5 \%)$ and routine $(5.6 \%)$. The majority of the patients presented with medical complaints $(n=364$, $58.5 \%)$ as opposed to trauma $(n=258,41.5 \%)$ (Table 1$)$.

The top five complaints reported were stab wounds (24.4\%), abdominal pain $(16.4 \%)$, dyspnoea $(3.9 \%)$, road traffic accidents (3.4\%) and chest pain (3.1\%). The top five final diagnoses at this hospital were injury or poisoning (38.9\%), infections and infestations (14.0\%), gastrointestinal disorders (10.3\%), nervous system disorders $(8.4 \%)$, and respiratory, thoracic and mediastinal disorders along with pregnancy, puerperium and perinatal conditions $(3.5 \%)$. Furthermore, of all trauma patients, the majority had stab/cut injuries (64.0\%), mainly in the extremities (35.3\%) (Table 2).

\section{Table 1. Characteristics of the patient population by site}

\begin{tabular}{|c|c|c|c|}
\hline Variable* & Frere Hospital $(N=2391), n(\%)$ & $\begin{array}{l}\text { Nelson Mandela Academic } \\
\text { Hospital }(N=622), n(\%)\end{array}$ & $\begin{array}{l}\text { Mthatha Regional Hospital } \\
(N=1258), n(\%)\end{array}$ \\
\hline \multicolumn{4}{|l|}{ Age (years) } \\
\hline $18-30$ & $992(41.5)$ & $203(32.6)$ & $504(40.1)$ \\
\hline $31-50$ & $929(38.9)$ & $112(18.0)$ & $393(31.2)$ \\
\hline $51-70$ & $352(14.7)$ & $115(18.5)$ & $230(18.3)$ \\
\hline$>70$ & $78(3.3)$ & $192(30.9)$ & $131(10.4)$ \\
\hline Not recorded & $38(1.6)$ & 0 & 0 \\
\hline$<18$ & $2(0.1)$ & 0 & 0 \\
\hline \multicolumn{4}{|l|}{ Sex } \\
\hline Male & $1360(56.9)$ & $375(60.2)$ & $539(42.8)$ \\
\hline Female & $994(41.6)$ & $247(39.8)$ & $719(57.2)$ \\
\hline Not recorded & $37(1.5)$ & 0 & 0 \\
\hline \multicolumn{4}{|l|}{ Arrival time } \\
\hline $09 \mathrm{~h} 00-17 \mathrm{~h} 00$ & $1073(44.9)$ & $233(37.5)$ & $594(47.2)$ \\
\hline After hours & $1277(53.4)$ & $389(62.5)$ & $663(52.7)$ \\
\hline Not recorded & $41(1.7)$ & 0 & $1(0.1)$ \\
\hline \multicolumn{4}{|l|}{ Arrival method } \\
\hline Self-transport & $1577(66.0)$ & $229(36.8)$ & $936(74.4)$ \\
\hline Ambulance & $742(31.0)$ & $389(62.5)$ & $301(23.9)$ \\
\hline Police & $15(0.6)$ & 0 & $10(0.79)$ \\
\hline Not recorded & $57(2.4)$ & $4(0.6)$ & $11(0.87)$ \\
\hline \multicolumn{4}{|l|}{ Referral status } \\
\hline New complaint & $2016(84.3)$ & $206(33.1)$ & $910(72.3)$ \\
\hline Return visit & $55(2.3)$ & $10(1.6)$ & $31(2.5)$ \\
\hline Referral & $279(11.7)$ & $406(65.3)$ & $310(27.1)$ \\
\hline Not recorded & $41(1.7)$ & 0 & $7(0.6)$ \\
\hline \multicolumn{4}{|l|}{ SATS (acuity level) } \\
\hline Emergency (red) & $19(0.8)$ & 0 & 0 \\
\hline Very urgent (orange) & 375 (15.7) & $186(29.9)$ & $358(28.5)$ \\
\hline Urgent (yellow) & $698(29.2)$ & $401(64.5)$ & $868(69.0)$ \\
\hline Routine (green) & $1140(47.7)$ & $35(5.6)$ & $32(2.5)$ \\
\hline Absconded (blue) & $1(0.04)$ & 0 & 0 \\
\hline Unknown & $158(6.6)$ & 0 & 0 \\
\hline \multicolumn{4}{|l|}{ Trauma v. medical } \\
\hline Trauma & $1656(69.3)$ & $258(41.5)$ & $344(27.3)$ \\
\hline Medical & $697(29.2)$ & $364(58.5)$ & $914(72.7)$ \\
\hline Not recorded & $38(1.6)$ & 0 & 0 \\
\hline
\end{tabular}




\begin{tabular}{|c|c|c|c|c|}
\hline Variable ${ }^{*}$ & $\begin{array}{l}\text { Frere Hospital } \\
(N=1656), n(\%)\end{array}$ & $\begin{array}{l}\text { Nelson Mandela } \\
\text { Academic Hospital } \\
(N=258), n(\%)\end{array}$ & $\begin{array}{l}\text { Mthatha Regional } \\
\text { Hospital }(N=344), n(\%)\end{array}$ & $\begin{array}{l}\text { Aggregate data } \\
(N=2258), n(\%)\end{array}$ \\
\hline \multicolumn{5}{|l|}{ Mechanism } \\
\hline Stab/cut & $494(29.8)$ & $165(64.0)$ & $91(26.5)$ & $750(33.2)$ \\
\hline Blunt force & $369(22.3)$ & $11(4.3)$ & $79(23.0)$ & $459(20.3)$ \\
\hline Fall & $367(22.2)$ & $20(7.8)$ & $51(14.8)$ & $438(19.4)$ \\
\hline Road traffic & $303(18.3)$ & $34(13.2)$ & $62(18.0)$ & $399(17.7)$ \\
\hline Animal/human bite & $37(2.2)$ & 0 & $24(7.0)$ & $61(2.7)$ \\
\hline Poisoning/ingestion & $25(1.5)$ & $5(1.9)$ & $10(2.9)$ & $40(1.8)$ \\
\hline Burn & $20(1.2)$ & $1(.39)$ & $13(3.8)$ & $34(1.5)$ \\
\hline Gunshot & $11(0.7)$ & $18(7.0)$ & $3(.87)$ & $32(1.4)$ \\
\hline Not recorded & $12(0.7)$ & $4(1.6)$ & $11(3.2)$ & $27(1.2)$ \\
\hline Drowning & $1(0.06)$ & 0 & 0 & $1(0.04)$ \\
\hline \multicolumn{5}{|l|}{ Location $^{\dagger}$} \\
\hline Extremity & $983(59.4)$ & $91(35.3)$ & $176(51.2)$ & $1250(55.4)$ \\
\hline Head/face/neck & $500(30.2)$ & $65(25.2)$ & $147(42.7)$ & $712(31.5)$ \\
\hline Back & $243(14.7)$ & $56(21.7)$ & $39(11.3)$ & $338(15.0)$ \\
\hline Chest & $99(6.0)$ & $74(28.7)$ & $21(6.1)$ & $194(8.6)$ \\
\hline Abdomen & $80(4.8)$ & $34(13.2)$ & $17(4.9)$ & $131(5.8)$ \\
\hline Eyes & $59(3.6)$ & $10(3.9)$ & $13(3.8)$ & $82(3.6)$ \\
\hline Pelvis & $41(2.5)$ & $7(2.7)$ & $14(4.1)$ & $62(2.7)$ \\
\hline Not recorded & $4(0.2)$ & 0 & 0 & $4(0.2)$ \\
\hline
\end{tabular}

\section{Mthatha Regional Hospital}

Of the 1258 patients enrolled at this site, most were female (719, $57.2 \%)$. The median age was 35 years (IQR $26-54$ ). Most patients visited the EC outside the normal hours of $09 \mathrm{~h} 00-17 \mathrm{~h} 00$ (663, $52.7 \%)$, largely with new complaints ( $n=910,72.3 \%)$, and arrived via self-transport $(n=936,74.4 \%)$. Patients were triaged as very urgent (28.5\%), urgent $(69.0 \%)$ and routine $(2.5 \%)$. More patients presented with medical complaints $(n=914,72.7 \%)$ than with trauma $(n=344$, 27.3\%) (Table 1).

The top five chief complaints reported were dyspnoea (9.7\%), abdominal pain (9.5\%), assault (7.6\%), stab wounds (4.7\%) and loss of energy (4.6\%). The top five final diagnoses were infections and infestations (25.8\%), injury or poisoning (23.5\%), nervous system disorders (8.5\%), gastrointestinal disorders (9.0\%), and pregnancy, puerperium and perinatal conditions (5.9\%). Furthermore, the majority of the trauma patients had stab/cut injuries (64.0\%), mainly in the extremities (35.3\%) (Table 2).

\section{Aggregate}

Across the three sites, of 4271 patients enrolled in the study, 52.8\% ( $n=2$ 258) presented with trauma (Table 2). Of these patients, 1459 (64.6\%) were males, 777 (34.4\%) females, and for 22 (1.0\%) sex was not recorded. Of the total patients, the majority were aged 18 30 years ( $n=1786,41.8 \%)$, of whom $62.1 \%$ ( $n=1109)$ presented with trauma.

The top five final diagnoses reported across the sites were injury or poisoning (42.5\%), infections and infestations (14.3\%), gastrointestinal disorders (6.5\%), musculoskeletal and connective tissue disorders (6.5\%) and nervous system disorders (6.0\%) (Fig. 2). Stratified by gender, the most frequent diagnoses were abdominal pain $(n=22,11.7 \%)$ for females and stab wounds for males $(n=397$, $17.5 \%)$.

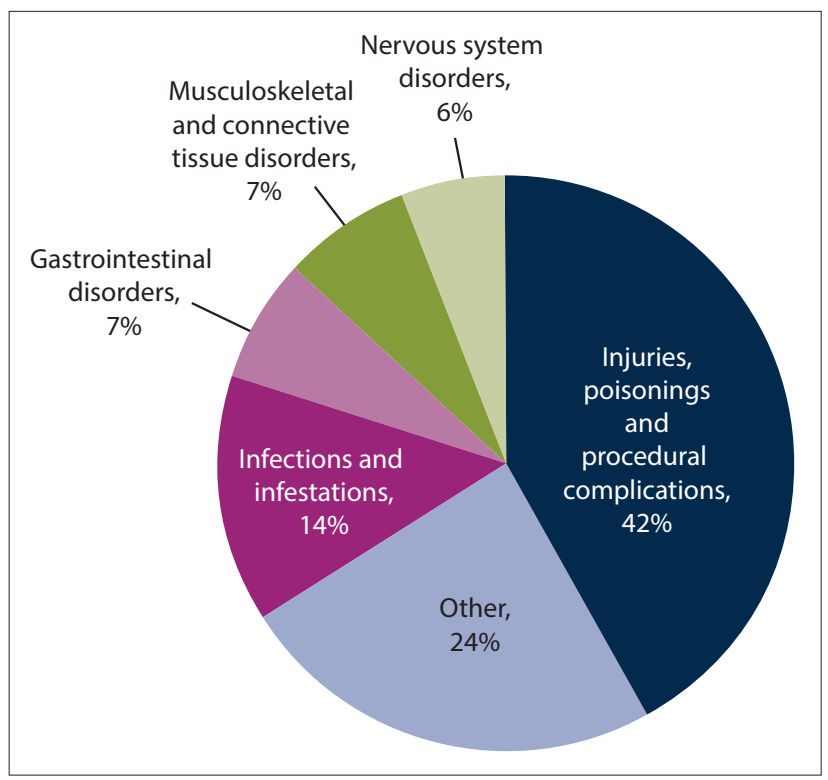

Fig. 2. Final diagnoses by system organ class for aggregate data. ('Other' category comprises respiratory, thoracic and mediastinal disorders $4.4 \%$, pregnancy, puerperium and perinatal conditions 3.1\%, general disorders and administration site conditions $2.6 \%$, metabolism and nutrition disorders $1.9 \%$, psychiatric disorders $1.9 \%$, cardiac disorders $1.7 \%$, vascular disorders $1.6 \%$, renal and urinary disorders $1.2 \%$, reproductive system and breast disorders $1.1 \%$, social circumstances $1.0 \%$, surgical and medical procedures $0.8 \%$, neoplasms - benign, malignant and unspecified $0.8 \%$, eye disorders $0.4 \%$, skin and subcutaneous tissue disorders $0.4 \%$, blood and lymphatic system disorders $0.4 \%$, hepatobiliary disorders $0.4 \%$, ear and labyrinth disorders $0.2 \%$, congenital, familial and genetic disorders $0.1 \%$, immune system disorders $0.1 \%$, investigations $0.1 \%$.) 


\section{Discussion}

SA has an injury death rate that is nearly twice the global average, in conjunction with a population burdened with chronic conditions, particularly HIV and tuberculosis. ${ }^{[7]}$ It is also one of the only countries where the death rates from intentional injuries exceed those from unintentional injuries. ${ }^{[8,9]}$ Our study demonstrated a high burden of trauma-related conditions in a mostly young male population.

Across all three study sites, the data illustrate that most patients utilising emergency services were aged $<30$ years and presented outside normal hours. Specifically, there was a higher proportion of male patients presenting with trauma in the two tertiary care centres of Frere Hospital and Nelson Mandela Academic Hospital than in the regional facility, which had a predominance of female patients with medical complaints. This gender disparity is reinforced by the most frequent EC diagnoses: stab wounds for males and abdominal pain for females. These differences across healthcare system levels could reflect a larger issue of vulnerable segments of the population such as young males who do not have routine access to or regularly use healthcare services. Instead, their initial exposure to the medical system is likely to be at a tertiary care centre when presenting with significant trauma.

Of the 4233 patients enrolled in this study, $52.8 \%$ presented to the EC with trauma-related injuries. This is much higher than in the neighbouring province of KwaZulu-Natal, where only $25 \%$ of the emergency workload is dedicated to trauma. ${ }^{[10]}$ Our findings are at odds with a retrospective chart review study that analysed reasons for visits to the ECs of Nelson Mandela Academic Hospital and Mthatha Regional Hospital from 1997 to 2000 and reported the incidence of trauma as $3.2 \% \cdot{ }^{[1]}$ It may be possible that either the amount of trauma in this area has risen significantly over the course of 20 years or the reporting structures previously used are inadequate to capture the burden of disease from trauma. In conjunction with the data from Frere Hospital in East London, where $70.3 \%$ of patients presented to the EC with trauma, it is clear that there is a need for prompt implementation of preventive strategies to mitigate the burden of disease from trauma in this region.

When looking at all patients presenting with trauma, the four primary mechanisms of injury observed were stab wounds (33.2\%), blunt force (20.2\%), falls (19.4\%) and road traffic accidents (17.7\%). This is consistent with findings in Cape Town, Western Cape Province, where a 16-month prospective study characterising trauma admissions at Groote Schuur Hospital from 2010 to 2011 found the same four leading mechanisms of injury of stab wounds (22.7\%), falls (18.7\%), blunt force (18.1\%) and road traffic accidents (16.0\%). ${ }^{[12]}$ The consistency in the major types of injury across provinces necessitates intervention.

Furthermore, we must keep in mind that a proportion of individuals who died of trauma-related causes would have been taken directly to the mortuary without reaching the healthcare sector. A recent study of the Transkei region used record analysis of 24693 medicolegal autopsies performed at Mthatha Forensic Pathology Laboratory from 1996 to 2015 to determine that the average rate of unnatural death is 205 per 100000 per year in this region, with the cause of death in two-thirds (69\%) of the cases being trauma. ${ }^{[13]}$ These figures serve to highlight that the burden of trauma in this area probably exceeds the conservative estimate suggested by our data and presses the need for better trauma care.

\section{Study limitations}

The present study was a secondary analysis of data from a larger study, so there are a number of limitations that need to be addressed.
The lack of electronic medical records and a uniform process by which patients entered the EC made it difficult to capture all patients who presented for care. We acknowledge the significant selection bias inherent in the fact that only patients who were enrolled in the HIV WISE study were included in this analysis. A significant proportion of patients were not captured, i.e. those who were too sick for HIV testing (SATS category 'red' or being resuscitated) or unable to consent for HIV testing (aged $<18$ years or presenting with altered mental status), or the HIV counsellors did not get to them (unable to be enrolled owing to limitations in staffing availability). Although the data encompass approximately one-third of the patients who presented to the three ECs during the study period, we believe that the large proportion of trauma documented (52\% of enrolled patients) can be considered representative of the remaining twothirds of the patient population who were not enrolled in the HIV study owing to individual preference, staffing constraints or unstable condition of the patient. This percentage is in fact likely to be an under-representation of the true burden of trauma in the EC because the HIV testing study excluded patients in a critical condition, which is often the result of trauma.

While we acknowledge the inherent flaws that result from insufficient enrolments, we found the high burden of trauma-related injury and the demographics of the patient population sufficiently striking to make a case to support trauma training in the Eastern Cape, and to indicate that the EC is a 'safety net' service that provides care to a large volume of young males and is therefore a potential setting for the implementation of trauma prevention interventions.

\section{Conclusions}

This study draws attention to the proportionally rising issue of trauma in the Eastern Cape region of SA. Trauma training is an important priority intervention that must be addressed. Furthermore, policymakers need to strategically develop interventions for trauma prevention and management in this region. In order to do so, further research specifically analysing the treatment needs of trauma patients in this region is of paramount importance in determining the extent of training required to adequately meet the needs of these trauma victims.

\section{Declaration. None.}

Acknowledgements. The authors thank the staff in the Frere Hospital, Nelson Mandela Academic Hospital and Mthatha Regional Hospital EDs for making this research possible, the WISE HIV counselling and testing team for their dedication and hard work during the study, and Nomazamo Mvandaba for her help on this project. We also thank Dr Thomas Quinn and the International HIV STD Laboratory at the National Institutes of Health (NIH), USA, for their support of the WISE study and ongoing collaboration at Walter Sisulu University.

Author contributions. $\mathrm{BH}$ conceived the original idea and designed the study protocol. KC, AR, VC and PM co-ordinated the study and data collection. $\mathrm{KC}$ and $\mathrm{AR}$ carried out analysis of data. $\mathrm{KC}$ prepared the manuscript. BH, AR, VC, FP, PM, JI, LW, TQ and RM provided substantial edits and revisions.

Funding. This research was supported by the South African Medical Research Council and the Division of Intramural Research, National Institute of Allergy and Infectious Diseases, NIH. The funders had no role in the study design, data collection and analysis, decision to publish, or preparation of the manuscript.

Conflicts of interest. None. 
1. Thind A, Hsia R, Mabweijano J, Hicks ER, Zakariah A, Mock CN. Prehospital and emergency care. In: Debas HT, Donkor P, Gawande A, Jamison DT, Kruk ME, Mock CN, eds. Essential Surgery: Disease Control Priorities. 3rd ed., vol. 1. Washington, DC: International Bank for Reconstruction and Development/World Bank, 2015:245.

2. Chang CY, Abujaber S, Reynolds TA, Camargo CA Jr, Obermeyer Z. Burden of emergency conditions and emergency care usage: New estimates from 40 countries. Emerg Med J 2016;33(11):794-800.
and and emergency care usage: New estimates from

3. Obermeyer Z, Abujaber S, Makar M, et al. Emergency care in 59 low-and middle-income countries: A systematic review. Bull World Health Organ 2015;93(8):577-586. https://doi.org/10.2471/

4. Mowafi H, Dworkis D, Bisanzo M, et al. Making recording and analysis of chief complaint a priority for global emergency care research in low-income countries. Acad Emerg Med 2013;20(12):1241-1245. https://doi.org/10.11

5. Reynolds TA, Calvello EJ, Broccoli MC, et al. AFEM consensus conference 2013 summary: Emergency care in Africa - where are we now? Afr J Emerg Med 2014;4(3):158-163. https://doi.org/10.1016/j. afjem.2014.07.00

6. Hansoti B, Stead D, Parrish A, et al. HIV testing in a South African emergency department: A missed opportunity. PloS One 2018;13(3):e0193858. https://doi.org/10.1371/journal.pone.0193858

7. John J, Matshoba W. Trauma care - the Eastern Cape story. S Afr Med J 2015;105(6):500. https://doi org/10.7196/SAMJ.9794

8. Schuurman N, Cinnamon J, Matzopoulos R, Fawcett V, Nicol A, Hameed SM. Collecting injury surveillance data in low- and middle-income countries: The Cape Town Trauma Registry pilot. Glob Public Health 2011;6(8):874-889. https://doi.org/10.1080/17441692.2010.516268
9. Statistics South Africa. Mortality and causes of death in South Africa: Findings from death notification. Pretoria: Stats SA, 2016. https://www.statssa.gov.za/publications/P03093/P030932016.pdf (accessed 30 June 2018).

10. Hardcastle TC, Oosthuizen G, Clarke D, Lutge E. Trauma, a preventable burden of disease in South Africa: Review of the evidence, with a focus on KwaZulu-Natal. South African Health Review 2016;2016(1):179-189. https://journals.co.za/docserver/fulltext/healthr/2016/1/healthr_2016_a17.pd f?expires=1574458830\&id=id\&accname=guest\&checksum=B25E37E87F2523DD3074E24687476585 (accessed 3 December 2019)

11. Dhaffala A, Longo-Mbenza B, Kingu J, et al. Demographic profile and epidemiology of injury in Mthatha, South Africa. Afr Health Sci 2013;13(4):1144-1148. https://doi.org/10.4314/ahs.v13i4.40

2. Schuurman N, Cinnamon J, Walker BB, et al. Intentional injury and violence in Cape Town, South Africa: An epidemiological analysis of trauma admissions data Glob Health Action 2015:8.27016. https//doi org/10.3402/gha.v8.27016

13. Meel BL. Incidence of unnatural deaths in Transkei sub-region of South Africa (1996 - 2015). S Afr Fam Pract 2017;59(4):138-142. https://doi.org/10.1080/20786190.2017.1292697

Accepted 29 July 2019 